\title{
High-Frequency Airway Oscillating Device for Respiratory Muscle Training in Subjects With COPD
}

\author{
Enya Daynes MSc, Neil J Greening PhD MD, Theresa C Harvey-Dunstan MSc, and \\ Sally J Singh PhD
}

\begin{abstract}
BACKGROUND: COPD is characterized by expiratory flow limitation, which results in symptomatic dyspnea and reduced exercise capacity. Changes in breathing mechanics mean the respiratory muscles are unable to respond to the ventilatory demands, increasing the sensation of dyspnea. A high-frequency oscillating device has been developed to improve dyspnea in patients with COPD. We conducted a feasibility trial to gain insight into the potential for recruitment, retention, and study design for a future randomized controlled trial. METHODS: Symptomatic subjects with COPD were included on the basis of a Medical Research Council (MRC) score $\geq 3$ and FEV $_{1} / F / C<0.70$ ). Patients were excluded if they received pulmonary rehabilitation within the last 6 months. The intervention employed the device for 8 weeks, 3 times daily. Clinical outcomes included the MRC score, maximal expiratory and inspiratory pressures $\left(\mathbf{P}_{\text {Emax }} / \mathbf{P}_{\text {Imax }}\right)$, the incremental shuttle walk test (ISWT), and the endurance shuttle walk test (ESWT). RESULTS: We successfully recruited 23 subjects with established COPD $(65.2 \%$ male, mean age $65 \pm 5.03$ y, mean \% predicted FEV $43.9 \pm 16$, mean $\mathrm{FEV}_{1} / \mathrm{FVC}$ ratio $0.46 \pm 0.13$, and median [interquartile range] MRC 4 [3-5]). There was a significant change in MRC from 4 to 3 pre to post intervention $(P=.003)$. There was a statistically significant difference in $P_{\text {Emax }} P<.008$ and $P_{\operatorname{Imax}} P=.044$. There were no significant differences observed in the ISWT or ESWT. CONCLUSIONS: This study design appeared feasible to proceed to a clinical effectiveness trial. The use of the device for 8 weeks showed a significant improvement in $\mathbf{P}_{\text {Emax }}, \mathbf{P}_{\text {Imax }}$, and reduced symptomatic dyspnea on the MRC dyspnea score. The results of this study should encourage a randomized controlled trial. Key words: breathing exercises; pulmonary disease; COPD; dyspnea; respiratory muscles; positive-pressure respiration. [Respir Care 2018;63(5):584-590. (C) 2018 Daedalus Enterprises]
\end{abstract}

\section{Introduction}

COPD is characterized by expiratory flow limitation, which results in excessive dyspnea and reduced exercise capacity. Dyspnea is a multidimensional symptom and may be a result of the muscles of respiration being unable to meet the demands of the mechanical load and capacity,

The authors are affiliated with the Centre for Exercise \& Rehabilitation Science, Leicester Respiratory Biomedical Research Unit, Glenfield Hospital, and the University of Leicester, Leicester, United Kingdom.

This study was funded by Actegy, who owns the rights of the Aerosure device used. Devices were provided by Actegy for the purpose of the trial. This study was also sponsored by the University Hospitals of Leicester NHS Trust.

The authors have disclosed no conflicts of interest. causing respiratory muscle dysfunction and the sensation of dyspnea. Longstanding dyspnea can result in reduced exercise capacity and impaired quality of life. ${ }^{1}$ A method of therapy for the management of dyspnea is inspiratory muscle training, which is frequently used in the COPD population. $^{2}$

The benefits of inspiratory muscle training have been identified in several randomized controlled trials and summarized in a systematic review by Gosselink et al. ${ }^{2}$ Key

\footnotetext{
Correspondence: Enya Daynes MSc, Centre for Exercise and Rehabilitation Science, Leicester Respiratory Biomedical Research Unit, Glenfield Hospital, Leicester LE3 9QP, United Kingdom. E-mail: enya.daynes@uhl-tr.nhs.uk.
}

DOI: $10.4187 /$ respcare. 05837 


\section{Respiratory Muscle Training IN COPD}

benefits are believed to be increased inspiratory muscle strength and endurance, functional exercise capacity, and health-related quality of life when compared to a control group; these improvements appear meaningful in terms of quality of life, dyspnea, and muscle strength. ${ }^{2-5}$ This metaanalysis explores randomized controlled trials using inspiratory muscle training programs at $\geq 30 \%$ of their maximum. In addition, high-intensity inspiratory muscle training, identified as training at $50 \%$ of a subject's maximum, has been shown to improve inspiratory muscle function in those with moderate to severe COPD. ${ }^{6}$ Research has also suggested that long-term inspiratory muscle training can decrease the use of health services and reduce hospital length of stay. ${ }^{7}$ Differences among training protocols and subsequent variances in results make this treatment difficult to implement and generalize.

Combined respiratory muscle training (RMT) is an additional technique for the management of dyspnea, although this therapy has been used less frequently despite the additional benefit of training expiratory muscles. It has been suggested that up to $50 \%$ of individuals with moderate to severe COPD exhibit expiratory muscle weakness in parallel with inspiratory muscles. ${ }^{8}$ While there is a limited evidence base for combined inspiratory and expiratory muscle training, it has been shown to improve respiratory muscle strength and endurance with an increase in the 6-min walk distance compared with inspiratory muscle training alone. ${ }^{9}$ Reference values for maximum expiratory pressure $\left(\mathrm{P}_{\text {Emax }}\right)$ have been discussed, but there has been no definitive categorization of expiratory muscle weakness. As a result it is difficult to identify patients who may benefit from combined respiratory muscle training. The mechanisms and effectiveness of RMT have been a topic for debate and are not fully accepted as a method to manage COPD. The joint American College of Chest Physicians/American Association of Cardiovascular and Pulmonary Rehabilitation Committee declared that a stimulus or load applied to the respiratory muscles during training is sufficient to augment respiratory muscle strength and is associated with increased exercise capacity and decreased dyspnea. However, the National Institute for Clinical Excellence does not yet recommend RMT in COPD management due to a disparity in the research. ${ }^{10,11}$

The Aerosure Medic (Aerosure, Bracknell, United Kingdom) is a flow-resistive device designed to offer resistance on inspiration and expiration with the aim to reduce dyspnea by improving breathing efficiency. The device also offers oscillations for mucociliary clearance, which may assist with reducing dyspnea by addressing air-flow obstruction. ${ }^{12}$ The combination of RMT and mucociliary clearance may contribute further to the management of dyspnea. This study assesses a number of outcomes including dyspnea, cough frequency, and sputum clearance to provide quantitative data on the use of a RMT device,

\section{QUICK LOOK}

\section{Current knowledge}

Respiratory muscle dysfunction can contribute to progressive dyspnea. Inspiratory muscle training has been shown to be effective in reducing dyspnea and improving inspiratory muscle strength in patients with COPD.

\section{What this paper contributes to our knowledge}

The use of high-frequency airway oscillations improved inspiratory and expiratory muscle strength resulting in an improvement in dyspnea. This should encourage a future randomized controlled trial.

which will inform and refine the potential for a clinical effectiveness trial. The objectives of this study were to (1) to assess the recruitment rate of participants and eligibility in regards to the inclusion and exclusion criteria and gain insight into dropout rates of the training program; (2) to explore device adherence and the training program; (3) to assess a number of outcomes and understand their feasibility in the use of a large clinical effectiveness trial; and (4) to explore and establish a primary outcome measure for the design of a clinical effectiveness trial and give insight into the sample size necessary.

\section{Methods}

This was a feasibility study designed to investigate the use of an RMT device to inform the clinical effectiveness of a randomized controlled trial. Ethical approval was obtained by the London Central National Health Service Health Research Authority and the Local Research Ethics Committee. The ISRCTN trial number is ISRCTN81979106.

\section{Participant Selection}

Twenty-four symptomatic subjects with COPD were recruited from the pulmonary rehabilitation database at the University Hospitals of Leicester. The sample size was pragmatic and was deemed to be appropriate to assess the feasibility of a clinical effectiveness trial. Subjects were included if they had stable COPD and a Medical Research Council (MRC) dyspnea score of $\geq 3$. Patients were excluded if they had completed pulmonary rehabilitation within the last 6 months. A diagnosis was confirmed by spirometry measures as outlined by the Global Initiative for Chronic Obstructive Lung Disease (GOLD) 2011 standards $\left(\mathrm{FEV}_{1} / \mathrm{FVC}\right.$ ratio $\left.<0.70\right){ }^{13}$ 


\section{Respiratory Muscle Training IN COPD}

The clinical outcomes for the study were the MRC dyspnea score, $\mathrm{P}_{\text {Emax }}$, and inspiratory pressure $\left(\mathrm{P}_{\text {Imax }}\right)$, performed in line with the American Thoracic Society/European Respiratory Society statement. ${ }^{14} \mathrm{P}_{\text {Emax }}$ was tested from total lung capacity, and $\mathrm{P}_{\text {Imax }}$ was tested from residual lung volume. Each test was performed in an upright sitting position. Subjects were instructed to perform the maneuver maximally and to sustain for a minimum of $2 \mathrm{~s}$. The test was performed a minimum of 4 times and up to 7 times if the subject continued to improve within the accepted tolerance, which was performed by a trained professional to optimize volitional effort. The incremental shuttle walk test (ISWT) and endurance shuttle walk test (ESWT) were performed according to the American Thoracic Society/European Respiratory Society guidelines, which include a familiarization ISWT to account for a learning effect. ${ }^{15}$ The COPD Assessment Test and the Chronic Respiratory Questionnaire were used as a healthrelated quality-of-life measure. The Leicester Cough Questionnaire, London Activity of Daily Living Questionnaire, and the Hospital Anxiety and Depression Scale were also used. Questionnaires were completed by each subject with supervision before and after intervention. A post-trial event was held to discuss the results and to secure feedback on the proposed future trial design.

\section{Device}

The intervention used a high-frequency oscillating device (Aerosure Medic, Actegy, Bracknell, United Kingdom), which provided flow resistance and oscillations. This was a battery-operated, dual-action device providing oscillations at $25 \mathrm{~Hz}$, and resistance was provided in relation to the subject's flow. Subjects were instructed to use the device for 5 min, 3 times per day, performing deep maximal breathing. The intervention was used for a period of 8 weeks with the use of a self-reported diary and weekly telephone calls to monitor adherence and manage any device-related issues. All subjects were instructed to use the device for the period of the intervention phase and were allowed to keep the device upon completion of the trial.

\section{Statistical Analysis}

Data were analyzed using SPSS version 24 (IBM, North Castle, New York). The Wilcoxon signed-rank test was used because the data were non-parametric for all variables. Subjects were considered adherent if they completed $75 \%$ of the training protocol, as recorded in the self-reported diary. $\mathrm{P}_{\text {Imax }}$ was considered weak if $>-60 \mathrm{~cm} \mathrm{H}_{2} \mathrm{O}$ as reported in the literature. ${ }^{2}$ Because no reference values are available for $\mathrm{P}_{\text {Emax }}$, this was calculated based on the Evans formula to calculate the lower limit of normal: $\mathrm{P}_{\text {Emax }}$ for males $=117-(0.83 \times$ age $) ; \mathrm{P}_{\mathrm{Emax}}$ for

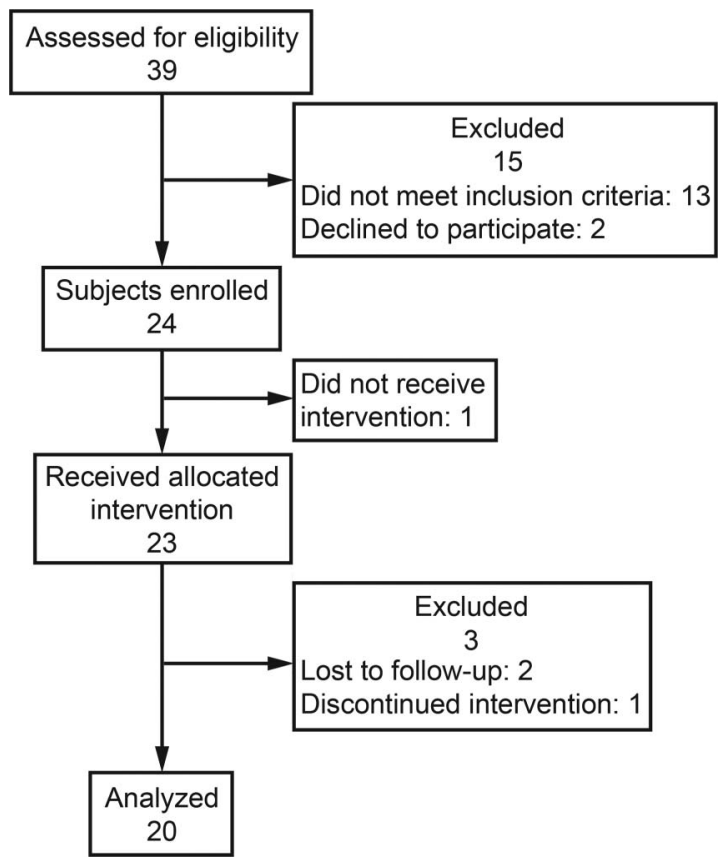

Fig. 1. Flow chart.

females $=95-(0.57 \times$ age $) .{ }^{16}$ Results were considered statistically significant if $P \leq .05$. A sub-group analysis was performed on subjects with poor inspiratory muscle strength $\left(>-60 \mathrm{~cm} \mathrm{H}_{2} \mathrm{O}\right)$ compared to those with normal inspiratory muscle strength $(<60 \mathrm{~cm}$ $\mathrm{H}_{2} \mathrm{O}$ ).

\section{Results}

Of 39 patients originally screened for eligibility, 24 $(61.5 \%)$ subjects were initially recruited. One subject was excluded after normal spirometry during the first visit. With 59\% of the initially identified patients being eligible for recruitment, the inclusion and exclusion criteria were deemed appropriate. There was a dropout rate of $13 \%$ and a self-reported adherence rate of $90 \%$ of subjects who met the minimum training requirement of $75 \%$ (Figure 1).

\section{Baseline Characteristics}

Baseline characteristics are shown in Table 1. The cohort was predominantly categorized as moderate, GOLD ${ }^{13}$ stage II, with a median MRC score of 4 (interquartile range $[\mathrm{IQR}] 3.00-4.75)$. Participants were managed with a combination of inhaled therapies, commonly triple therapy (ie, a combination of long-acting muscarinic antagonist, long-acting $\beta$ agonist, and inhaled corticosteroids). Mean (SD) $\mathrm{P}_{\text {Imax }}$ values were $-57.48 \pm 26 \mathrm{~cm} \mathrm{H}_{2} \mathrm{O}\left(\mathrm{P}_{\text {Imax }} \geq-60 \mathrm{~cm}\right.$ $\mathrm{H}_{2} \mathrm{O}$ qualified as inspiratory muscle weakness). ${ }^{2}$ Based on the Evans calculations, 7 subjects demonstrated expiratory 


\section{Respiratory Muscle Training IN COPD}

Table 1. Baseline Characteristics

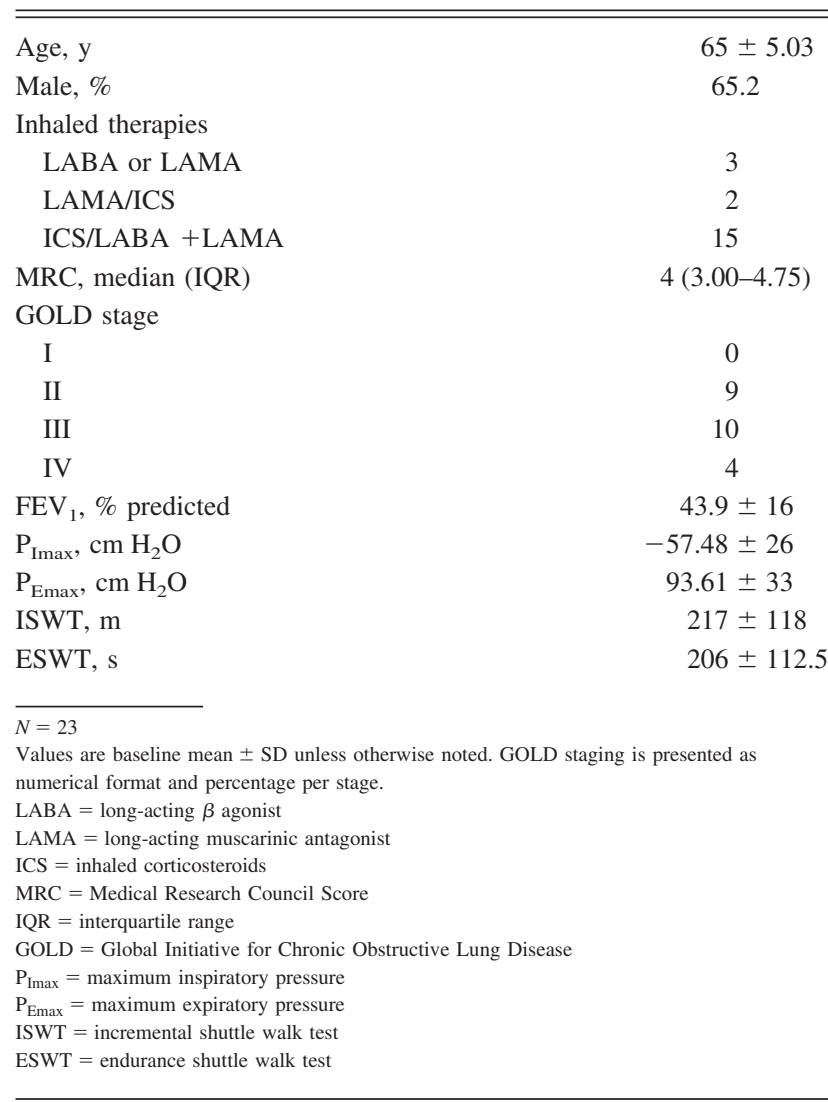

muscle weakness ranging from $41.33 \%$ to $88.64 \%$ of predicted values. ${ }^{16}$

Of the 23 enrolled subjects, 1 subject was withdrawn due to ill health, 1 subject was withdrawn for social reasons, and 1 subject stopped using the device due to an adverse event where the participant experienced unexplained vocal cord irritation. Therefore, 20 subjects were included in the analysis.

\section{Intervention}

After the 8-week intervention, data were collected on 20 subjects. Exercise test and respiratory muscle function data were completed on 19 of the participants. Overall, subjects improved their MRC score from 4 to 3 ( $P=.003$, 95\% CI 2.68-3.32). Subjects' median (IQR) $\mathrm{P}_{\mathrm{Imax}}$ improved from $-59 \mathrm{~cm} \mathrm{H}_{2} \mathrm{O}$ (34-74) to $-63 \mathrm{~cm} \mathrm{H}_{2} \mathrm{O}$ (4285) $(P=.044) . \mathrm{P}_{\mathrm{Emax}}$ improved from a median (IQR) of $102(62-125)$ to $110(97-137)(P<.003)$, which was statistically significant. No statistical or clinically meaningful change in exercise performance (ISWT or ESWT), Chronic Respiratory Questionnaire, Hospital Anxiety and Depression Scale, or London Activity of Daily Living Questionnaire were observed. A change in COPD
Table 2. Pre and Post Intervention

\begin{tabular}{|c|c|c|c|}
\hline & $\begin{array}{l}\text { Pre Intervention, } \\
\text { Median (IQR) }\end{array}$ & $\begin{array}{l}\text { Post Intervention, } \\
\text { Median (IQR) }\end{array}$ & $P$ \\
\hline MRC & $4(3-5)$ & $3(3-3)$ & .003 \\
\hline$+\mathrm{P}_{\text {Imax }}, \mathrm{cm} \mathrm{H}_{2} \mathrm{O}$ & $-59(34-74)$ & $-63(42-85)$ & .044 \\
\hline$+\mathrm{P}_{\mathrm{Emax}}, \mathrm{cm} \mathrm{H}_{2} \mathrm{O}$ & $102(62-125)$ & $110(97-137)$ & .008 \\
\hline +ISWT, m & $200(140-260)$ & $240(170-270)$ & .68 \\
\hline +ESWT, s & $170.5(130.5-246.75)$ & $203(142.25-274.25)$ & .51 \\
\hline CRQ dyspnea & $2.6(2.0-2.8)$ & $2.5(2.05-3.70)$ & .32 \\
\hline CRQ total & $16.97(12.91-18.12)$ & $16.7(14.77-19.63)$ & .85 \\
\hline LCQ total & $15.71(12.66-19.36)$ & $21.5(16.25-25.50)$ & .14 \\
\hline HADS anxiety & $6(3-10)$ & $6(3.25-11.25)$ & .24 \\
\hline HADS depression & $6(4-10)$ & $5(4-7.5)$ & .19 \\
\hline LCADL total & $32(28-45)$ & $29(23.25-39)$ & .26 \\
\hline CAT total & $24(18-29)$ & $21.5(16.25-25.5)$ & .14 \\
\hline CAT sputum & $3(2-4)$ & $3(2-3.75)$ & .76 \\
\hline $\begin{array}{l}N=20,+\mathrm{N}=19 \\
\mathrm{IQR}=\text { interquartile range } \\
\text { MRC }=\text { Medical Researcl } \\
\mathrm{P}_{\text {Imax }}=\text { maximum inspira } \\
\mathrm{P}_{\text {Emax }}=\text { maximum expira } \\
\text { ISWT }=\text { incremental shut } \\
\text { ESWT }=\text { endurance shutt } \\
\text { CRQ }=\text { Chronic Respirat } \\
\text { LCQ }=\text { Leicester Cough } \\
\text { HADS = Hospital Anxiet } \\
\text { LCADL = London Chest } \\
\text { CAT }=\text { COPD assessmen }\end{array}$ & $\begin{array}{l}\text { h Council Score } \\
\text { atory pressure } \\
\text { atory pressure } \\
\text { ttle walking test } \\
\text { tle walking test } \\
\text { ory Questionnaire } \\
\text { Questionnaire } \\
\text { ty and Depression Score } \\
\text { Activity of Daily Living } \\
\text { tt test }\end{array}$ & & \\
\hline
\end{tabular}

Assessment Test score was seen (mean difference $=0.95 \pm 4.62)$ but this was not clinically meaningful (Table 2). ${ }^{17}$ Sub-group analysis demonstrated a greater improvement in $\mathrm{P}_{\text {Imax }}$ and ISWT in those with inspiratory muscle weakness $\left(>-60 \mathrm{~cm} \mathrm{H}_{2} \mathrm{O}\right)$ (Figure 2) (Table 3).

\section{Discussion}

The results of this study demonstrate appropriate eligibility criteria, a low dropout rate, and high adherence to the training protocol; we therefore believe that this study design is appropriate and a larger randomized, controlled trial is deemed feasible. This study design demonstrates a high enrollment rate; while most potentially eligible participants were screened out due to current exacerbation, these patients were enthusiastic about treatment and it is likely that with an extended recruitment period we may be able to enroll these patients when they are stable (ie, no exacerbation in the last 4 weeks). The retention rate was high, with a $13 \%$ dropout rate, which is equal to or less than the attrition of similar study designs, suggesting that the training program was not too burdensome.

The use of the device for 8 weeks resulted in significant improvement in respiratory muscle strength and dyspnea, demonstrated by inspiratory and expiratory pressures and reduced MRC score, despite not intentionally recruiting 


\section{Respiratory Muscle Training IN COPD}
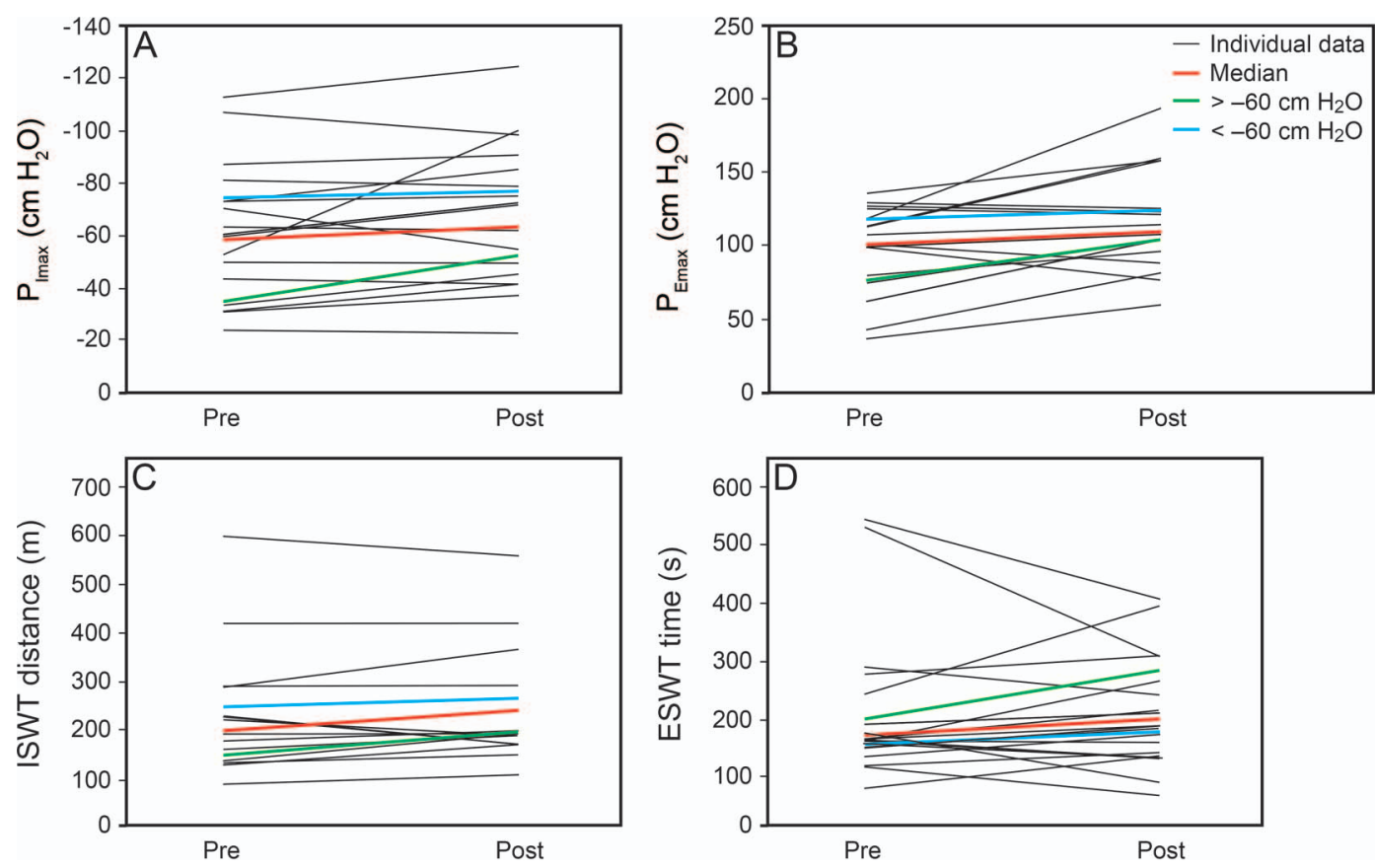

Fig. 2. A: $P_{I \max }$ inspiratory muscle pressures measured at the mouth. B: $P_{E \max }$ expiratory muscle pressures measured at the mouth. C: Incremental shuttle walk test (ISWT) measured pre- and post-8 week intervention phase. D: Endurance shuttle walk test (ESWT) measured pre- and post-8 week intervention phase.

Table 3. Comparison Between Participants Classified With Inspiratory Muscle weakness

\begin{tabular}{|c|c|c|c|c|c|c|}
\hline & \multicolumn{3}{|c|}{$\begin{array}{c}\text { Poor Inspiratory Muscle } \\
\text { Strength }\left(>-60 \mathrm{~cm} \mathrm{H}_{2} \mathrm{O}\right), n=9\end{array}$} & \multicolumn{3}{|c|}{$\begin{array}{l}\text { Normal Inspiratory Muscle } \\
\text { Strength }\left(<-60 \mathrm{~cm} \mathrm{H}_{2} \mathrm{O}\right), n=10\end{array}$} \\
\hline & $\begin{array}{l}\text { Pre Intervention, } \\
\text { Median (IQR) }\end{array}$ & $\begin{array}{l}\text { Post Intervention, } \\
\text { Median (IQR) }\end{array}$ & $P$ & $\begin{array}{l}\text { Pre Intervention, } \\
\text { Median (IQR) }\end{array}$ & $\begin{array}{l}\text { Post Intervention, } \\
\text { Median (IQR) }\end{array}$ & $P$ \\
\hline MRC & $4(3-5)$ & $3(3-3)$ & .02 & $4(3-4)$ & $3(2-3)$ & .058 \\
\hline $\mathrm{P}_{\text {Imax }}, \mathrm{cm} \mathrm{H}_{2} \mathrm{O}$ & $-35(31-49)$ & $-42(39-56)$ & .050 & $-74(63-86)$ & $-77(70-93)$ & .14 \\
\hline $\mathrm{P}_{\text {Emax }}, \mathrm{cm} \mathrm{H}_{2} \mathrm{O}$ & $77(57-102)$ & $105(83-111)$ & .36 & $118(108-134)$ & $125(109-159)$ & .059 \\
\hline ISWT, m & $150(100-245)$ & $200(160-260)$ & .064 & $250(180-290)$ & $265(185-283)$ & .44 \\
\hline ESWT, s & $200(120-278)$ & 285 (104-376) & .67 & $156(134-190)$ & 179 (144-224) & .11 \\
\hline
\end{tabular}

MRC $=$ Medical Research Council Score

$\mathrm{P}_{\operatorname{Imax}}=$ maximum inspiratory pressure

$\mathrm{P}_{\text {Emax }}=$ maximum expiratory pressure

ISWT $=$ incremental shuttle walking test

ESWT $=$ endurance shuttle walking test

participants with respiratory muscle weakness. Subjects with reduced inspiratory muscle strength demonstrated a greater improvement in dyspnea and respiratory muscle strength. While we observed an improvement in the ISWT and the ESWT, this was not statistically significant. There was a notable difference between GOLD severity and MRC dyspnea score at baseline, but as a result of the subjectivity of dyspnea and the inability of the MRC score to capture its complexity, this is unsurprising. ${ }^{18}$

The public and subject involvement allowed for insights into the training intensity and use of the device as well as a detailed understanding of how this affected dyspnea. It was useful to assist with the design of a clinical effectiveness trial and identified areas that had to be addressed (eg, diary cards, training intensity, and follow-up calls). A power calculation was performed based on the change in COPD Assessment Test score, and 84 subjects will be required for a randomized controlled trial. All outcome measures were deemed appropriate by the subjects to assess the effects of the device.

Dyspnea is the most common complaint for patients with COPD. ${ }^{1}$ It has been reported that respiratory muscle weakness is uncommon in patients with COPD, however the function of these subjects demonstrated that both in- 


\section{Respiratory Muscle Training IN COPD}

spiratory and expiratory muscles can be classified as weak, similar to findings of a number of other trials exploring RMT in COPD. ${ }^{2}$ This study did not select subjects on the basis of respiratory muscle weakness, but sub-group analysis indicates that those classified as weak had greater improvements in inspiratory muscle strength and ISWT, and both groups significantly improved dyspnea scores, therefore both groups will be included in the randomized controlled trial. Furthermore, the threshold for weakness appears to be arbitrary and does not account for an increased ventilatory demand as associated with patients with COPD. In addition, inspiratory muscle weakness has been associated with hyperinflation-induced diaphragm shortening and fiber shift toward oxidative type- 1 fibers in the diaphragm of patients with COPD. ${ }^{8}$ Although less is understood regarding expiratory muscle weakness, it has been suggested that $50 \%$ of patients with moderate to severe COPD exhibit expiratory muscle weakness in parallel with inspiratory muscles. ${ }^{2}$ Reference values for $\mathrm{P}_{\mathrm{Emax}}$ have been discussed, although there is no definitive conclusion of values that categorize expiratory muscle weakness.

The implementation of RMT is inconsistently applied in the COPD population, and there is a need for rigorous trials investigating this treatment. ${ }^{2}$ There is a large body of evidence for the evaluation of inspiratory muscle training, but differences in training protocols make it difficult to reach consensus, which limits clinical application. Expiratory muscle training has been shown to improve respiratory function when used in combination with inspiratory muscle training or oscillation for patients with mild to severe COPD. ${ }^{19}$ In this study, we proposed an 8-week training program of combined inspiratory and expiratory muscle training using a device 3 times per day to provide flow resistance and additional oscillations.

We did not see an important increase in exercise capacity, which conflicts with other research; however, this device was not used in conjunction with any other form of training or pulmonary rehabilitation, which may explain the results. It is possible that these participants saw improvements in dyspnea and respiratory muscle strength that did not translate into increased exercise capacity but may have improved the quality of exercise, which was not measured in this study. There are numerous other factors that may limit exercise capacity, such as reduced energy supply, leg discomfort, or comorbidities such as osteoarthritis, which may affect exercise capacity over time and influence the results. ${ }^{20}$ There were 2 recorded exacerbations during the follow-up period, which also may have affected exercise capacity.

\section{Limitations}

This single-arm study was devised to explore the feasibility of using an RMT device with COPD. This study has a small sample size and therefore is not generalizable to the COPD population; this research, however, has shown a reduction in dyspnea. This single-arm, non-blinded study may be subject to bias. In the absence of a control group, it is not possible to make any comment on clinical effectiveness at this stage because we are unable to rule out the potential of a placebo effect. While sub-group analysis may allow for some indication of population groups that may benefit from this device, this should be interpreted with caution due to the small sample size.

The device used in this study does not have the function to increase or reduce resistance and therefore may not be suitable for all patients with COPD; however, the device was accepted by the subjects and does not allow the subjects to make adjustments outside of the protocol.

\section{Conclusions}

The aim of this study was to explore the feasibility of designing a clinical effectiveness trial investigating the use of a high-frequency oscillation device in the management of dyspnea in COPD. The eligibility criteria enabled a suitable recruitment rate with a dropout of $13 \%$, which included subjects who were unable to complete the follow-up testing. The training intensity seems feasible with an adherence rate of $90 \%$ among subjects. Subjects of this cohort demonstrated a significant increase in $\mathrm{P}_{\text {Emax }}$ and $\mathrm{P}_{\text {Imax }}$ as well as a subsequent statistically significant reduction in dyspnea. This study provides encouraging results in reducing dyspnea with combined RMT with Aerosure device.

Outcome measures assessed the identified implications of using the device and therefore will assist with the future trial design. Public and subject involvement identified areas that may need to be addressed prior to a large clinical effectiveness trial. The results of this study should encourage a full randomized controlled trial.

\section{REFERENCES}

1. Laghi F, Tobin MJ. Disorders of the respiratory muscles. Am J Respir Crit Care Med 2003;168(1):10-48.

2. Gosselink R, De Vos J, Heuvel S, Segers J, Decramer M, Kwakkel G. Impact on inspiratory muscle training in patients with COPD: what is the evidence? Eur Respir J 2011;37:416-425.

3. Petrovic M, Reiter M, Zipko H, Pohl W, Wanke T. Effects of inspiratory muscle training on dynamic hyperinflation in patients with COPD. Int J Chron Obstruct Pulmon Dis 2012;7:797-805.

4. Sykes K, Hang HW. Inspiratory muscle training in the treatment of chronic obstructive pulmonary disease: randomized controlled trial. Am J Recreat Therap 2005;4(2):39-48.

5. Weiner P, Magadle R, Beckerman M, Weiner M, Berar-Yanay N. Maintenance of inspiratory muscle training in COPD patients: one year follow-up. Eur Respir J 2004;23(1):61-65.

6. Hill K, Jenkins SC, Philippe DL, Cecins N, Shepherd KL, Green DJ, et al. High-intensity inspiratory muscle training in COPD. Eur Respir J 2006;27(6):1119-1128. 


\section{Respiratory Muscle Training IN COPD}

7. Beckerman M, Magadle R, Weiner M, Weiner P. The effects of 1 year of specific inspiratory muscle training in patients with COPD. Chest 2005;128(5):3177-3182.

8. Ottenheijm CA, Heunks LM, Dekhuijzen PN. Diaphragm muscle fiber dysfunction in chronic obstructive pulmonary disease: toward a pathophysiological concept. Am J Respir Crit Care Med 2007; 175(12):1233-1240

9. Weiner P, Magadle R, Beckerman M, Weiner M, Berar-Yanay N. Comparison of specific expiratory, inspiratory, and combined muscle training programs in COPD. Chest 2003;124(4):1357-1364.

10. Pulmonary rehabilitation: joint ACCP/AACVPR evidence-based guidelines. ACCP/AACVPR Pulmonary Rehabilitation Guidelines Panel. American College of Chest Physicians. American Association of Cardiovascular and Pulmonary Rehabilitation. Chest 1997; 112(5):1363-1396

11. Chronic obstructive pulmonary disease in over 16s: diagnosis and management (CG101). London, UK: National Institute for Health Care Excellence; 2010.

12. Hristara-Papadopoulou A, Tsanakas J, Diomou G, Papadopoulou O. Current devices of respiratory physiotherapy. Hippokratia 2008;12(4): 211-220.

13. Global Initiative for Chronic Obstructive Lung Disease. Global strategy for the diagnosis, management and prevention of COPD 2017. http:// goldcopd.org/gold-2017-global-strategy-diagnosismanagementprevention-copd. Accessed January 9, 2018.
14. American Thoracic Society/European Respiratory Society. ATS/ERS Statement on respiratory muscle testing. Am J Respir Crit Care Med 2002;166(4):518-624.

15. Holland AE, Spruit MA, Troosters T, Puhan MA, Pepin V, Saey D, et al. An official European Respiratory Society/American Thoracic Society technical standard: field walking tests in chronic respiratory disease. Eur Respir J 2014;44(6):1428-1446.

16. Evans JA, Whitelaw WA. The assessment of maximal respiratory mouth pressures in adults. Respir Care 2009;54(10):1348-1359.

17. Kon SS, Canavan JL, Jones SE, Nolan CM, Clark AL, Dickson MJ, et al. Minimum clinically important difference for the COPD Assessment Test: a prospective analysis. Lancet Respir Med 2014;2(3): 195-203.

18. Lopez-Campos JL, Fernandez-Villar A, Calero-Acuña C, Represas-Represas C, Lopez-Ramírez C, Fernández VL, et al. Evaluation of the COPD Assessment Test and GOLD patient types: a cross-sectional analysis. Int J Chron Obstruct Pulmon Dis 2015; 10:975-984.

19. Battaglia E, Fulgenzi A, Ferrero ME. Rationale of the combined use of inspiratory and expiratory devices in improving maximal inspiratory pressure and maximal expiratory pressure of patients with chronic obstructive pulmonary disease. Arch Phys Med Rehabil 2009;90(6): 913-918.

20. Vogiatzis I, Zakynthinos G, Andrianopoulos V. Mechanisms of physical activity limitation in chronic lung diseases. Pulm Med 2012; 2012:634761. 